\title{
Fisiología reproductiva del pez Betta splendens en condiciones de laboratorio, Piedemonte Andino Amazónico (Colombia)
}

\author{
Murcia-Ordoñez, B.; Chaves, L.C.; España, W.F.; Castañeda, D.; Andrade, J. \\ Grupo de Investigación en Biodiversidad y Desarrollo Amazónico (BYDA), Universidad de la Amazonia, \\ Calle 17-Diagonal 17 con Carrera 3F, Barrio Porvenir, Florencia-Caquetá-Colombia. \\ E-mail: b.murcia@udla.edu.co
}

\begin{abstract}
Resumen
Murcia-Ordoñez, B.; Chaves, L.C.; España, W.F.; Castañeda, D.; Andrade, J.: Fisiología reproductiva del pez Betta splendens en condiciones de laboratorio, Piedemonte Andino Amazónico (Colombia). Rev. vet. 27: 2, 124-129, 2016. Las estrategias reproductivas (cortejo, fecundación, fertilización e incubación) del pez Betta splendens, así como su desarrollo embrionario (cigoto, mórula, blástula, gástrula, organogénesis y eclosión) fueron observados y registrados en 100 huevos previamente fecundados bajo condiciones de laboratorio (temperatura $27,8^{\circ} \mathrm{C}, \mathrm{pH} 6,8$ ) en la Universidad de la Amazonia (Florencia, Caquetá, Colombia), con el fin de obtener información que genere ventajas para la producción y comercialización de esta especie. Se utilizó el programa Toup View versión 2.1 con el cual se midieron las estructuras morfológicas de 32 ovocitos cada 5 minutos hasta la organogénesis y después cada cuatro horas hasta la eclosión. Se obtuvo una tasa de fertilidad de los desoves de $83 \pm 2,0 \%$. El tiempo transcurrido desde la fecundación hasta la eclosión fue de $36,13 \mathrm{~h}$. El tamaño del disco germinal fue de 529,39 $\mu \mathrm{m}$, el radio de 5,64 $\mu \mathrm{m}$ y el saco vitelino de 679,43 $\mu \mathrm{m}$. Después de la fecundación, el huevo sufrió una serie de cambios muy rápidos ( $\pm 36 \mathrm{~h})$ del desarrollo embrionario, incluyendo la segmentación (40 min), ciclaje (50 min), gastrulación (55 min), segmentación y organogénesis (16 min), morfogénesis $(21$ h), eclosión y estado larvario (13 h). Se concluye que el desarrollo embrionario de B. splendens es más corto (36.13 h) que el de otros peces teleósteos, dato que servirá como información base para estudios encauzados a la reproducción de esta especie con fines comerciales.
\end{abstract}

Palabras claves: pez Betta splendens, estrategias reproductivas, desarrollo embrionario.

\begin{abstract}
Murcia-Ordoñez, B.; Chaves, L.C.; España, W.F.; Castañeda, D.; Andrade, J.: Reproductive physiology Betta splendens fish in laboratory conditions, Piedemonte Andino Amazon (Colombia). Rev. vet. 27: 2, 124-129, 2016. It was observed and described the reproductive strategies (courtship process, fecundation, fertilization and incubation) and the embryo development (zygote, morula, blastula, gastrula, and organogenesis hatching) in 100 fertilized eggs of Betta splendens under laboratory conditions (temperature $27.8^{\circ} \mathrm{C}, \mathrm{pH} 6.8$ ) at Universidad de la Amazonia (Florencia, Caquetá, Colombia), in order to get information that generates advantages for the production and marketing of this species. Toup View program 2.1 version was used to measure the morphological structures of 32 oocytes every 5 minutes until organogenesis and then every four hours until hatching. It was obtained a percentage of fertility spawns of $83 \pm 2.0 \%$ and elapsed time (from fertilization to hatching) of $36.13 \mathrm{~h}$ for this species. Germinal disc size was $529.39 \mu \mathrm{m}$ with a radius of $5.64 \mu \mathrm{m}$ and a yolk sac 679,43 $\mu \mathrm{m}$. The egg undergoes a series of rapid changes $( \pm 36 \mathrm{~h})$ after fertilization generating embryonic development: segmentation (40 min), cycling (50 min), gastrulation (55 minutes), segmentation and organogenesis (16 min), morphogenesis (21 hours), hatching and larval stage (13 hours). It is concluded that $B$. splendens is a fish with a type of embryonic development fairly short $(36.13 \mathrm{~h})$ compared to the period presented in other teleost fish, data that will be useful for further studies related to the reproduction of this species for commercial purposes.
\end{abstract}

Key words: fish Betta splendens, reproductive strategies, embryonic development. 


\section{INTRODUCCIÓN}

Uno de los aspectos fisiológicos en los animales es el control de la reproducción que en peces involucra un proceso multifactorial donde interaccionan agentes ambientales (fotoperíodo, temperatura, oxígeno disuelto, salinidad, entre otros), sociales (comportamiento, preferencias reproductivas, presencia de otros individuos, densidad de población, proporción de sexos), neurales (sistemas sensoriales y receptores específicos), endocrinos (melatonina) ${ }^{8} \mathrm{y}$ nutricionales (preferencias tróficas) ${ }^{21}$ que no solo permiten generar estrategias reproductivas (colas amplias, policromía, rituales de cortejo, cantidad de huevos en postura, cuidado parental) ${ }^{24}$ sino su desarrollo embrionario (estadios embrionarios, larvarios y tiempos de ocurrencia) ${ }^{5}$.

A pesar de que los cambios ambientales afectan profundamente la función reproductiva en los peces teleósteos y que este hecho ocurre por alteraciones en el eje cerebro-hipófisis-gónadas, encargado de que la reproducción tenga lugar en el momento más favorable para la supervivencia de la progenie ${ }^{8}$, el vínculo entre el medio ambiente y la cascada neuroendocrina queda por aclarar $^{2,15,18,29}$.

Los estudios sobre reproducción de peces con fines comerciales se incrementan cada día más, principalmente los relacionados a los primeros estadios vitales (huevos, larvas y etapas tempranas de juveniles) ya que son críticos para las fluctuaciones en la abundancia de las poblaciones de peces explotadas ${ }^{33}$, como en el caso de Betta splendens, apetecido comercialmente ${ }^{26}$ por su estructura faríngea (laberinto) que le permite tomar oxígeno atmosférico cada 3 minutos aproximadamente ${ }^{3}$, dimorfismo sexual, (policromía) y el cuidado parental del macho en las primeras fases de desarrollo (fertilización a eclosión) ${ }^{12}$. Según algunos estudios, este pez genera -mediante la fertilización- una nube de semen o espuma que contiene componentes bactericidas y sustancias que modifican favorablemente la composición química del agua en derredor a los huevos, permitiendo así el desarrollo favorable del embrión y su eclosión ${ }^{3,17}$.

A pesar de ello, el tiempo de desarrollo así como las características embrionarias de $B$. splendens son poco conocidas, por lo cual resulta necesario profundizar conocimientos acerca de sus estrategias reproductivas (proceso de cortejo, fecundación, fertilización e incubación), así como conocer su desarrollo embrionario, datos que aportarían ventajas para su producción y comercialización.

\section{MATERIAL Y MÉTODOS}

El estudio se llevó a cabo en los laboratorios de investigación en fisiología animal e ictiología de la Universidad de la Amazonia (Florencia, Caquetá, Colombia), localizados a $1^{\circ} 36^{\prime} 25.95 \mathrm{~N}$ y $75^{\circ} 36^{\prime} 27.12 \mathrm{~W}$, con una altitud de $350 \mathrm{msnm}$., temperatura promedio de $25^{\circ} \mathrm{C}$ y humedad relativa del $80 \%$.
Se tomaron 100 huevos previamente fecundados de cuatro parejas de $B$. splendens sexualmente maduras, que fueron mantenidos en acuarios acorde a procedimientos habituales para esta especie $3,14,22,30$, incluyendo la observación y descripción de su estrategia reproductiva (procesos de cortejo, fecundación, fertilización e incubación).

Para la descripción de los cambios morfométricosy embriológicos se extrajo una muestra equivalente al $33 \%$ de ovocitos fecundados ${ }^{31}$, los cuales fueron clarificados (solución transparentadora YBAG/85) y fijados $\left(1000 \mathrm{ml} \mathrm{H}_{2} \mathrm{O}+6,5 \mathrm{~g} \mathrm{NaCl}+10 \mathrm{ml} \text { de formol } 40 \%\right)^{10}$ para su observación. Las muestras fueron colectadas cada 5 min hasta la evidencia de la organogénesis; después se colectaron cada $4 \mathrm{~h}$ hasta su eclosión, además se tomaron cuatro larvas para determinar aspectos morfométricos ${ }^{32}$. Se realizó la clasificación y descripción de los diferentes estadios ${ }^{25}$, así como la morfometría y merística de los huevos y larvas ${ }^{35}$; se separaron los estadios en pre-larvas, larvas y post-larvas ${ }^{20,25}$.

Las abreviaturas empleadas en este manuscrito son: minutos post-fecundación (MPF), diámetro del huevo (DH, medido a través de la distancia de la línea media horizontal del huevo, se nombra así cuando no hay diferencia entre la longitud y ancho del huevo); diámetro del saco vitelino (DSV= distancia de la línea media horizontal del vitelo), espacio perivitelino $(E P V=$ diferencia entre DH y DSV), longitud del vitelo ( $\mathrm{LV}=$ distancia entre márgenes anterior y posterior del saco vitelino), ancho del vitelo $(\mathrm{AV}=$ distancia entre márgenes dorsal y ventral del saco vitelino), distancia postorbital (DPO= distancia entre el borde del hocico y el margen anterior de las expansiones alares), eje del embrión ( $\mathrm{EE}=$ longitud desde la cabeza hasta su polo opuesto); longitud notocordal ( $\mathrm{LN}=$ distancia entre el extremo anterior de la cabeza hasta el final de la notocorda), ancho de notocordio (AN), largo de la cola (LC), ancho de vesícula óptica (AVO), largo vesícula óptica (LVO), plano de división (PD), blastómeros (B), polo animal (PA), polo vegetal (PV), discoblástula (DB), borde periblasto (BP), parte anterior (A), parte caudal (PC), vesícula de Kupffer (K), saco vitelino (SV), vitelo (V), vesícula óptica (VO), vesícula ótica (VOT), notocordia $(\mathrm{N})$, somitas (SM), cristalino (C), ubicación del corazón (CZ).

Todas las medidas fueron registradas con una cámara de video profesional USB $(1280 \times 1024 \mathrm{X})$ dispuesta en un estereomicroscopio (Zeiss Stemi 2000C) y analizadas con un programa computacional analizador de imágenes (Toup View versión 2.1 vimicro USB $2.0 \mathrm{UVC}$ ).

\section{RESULTADOS}

Proceso de cortejo y fertilización. Se inició con la formación del nido por parte del macho (Figura 1a), el cual segregó una sustancia blanca en forma de espuma que se mantuvo sólida y consistente en la superficie del acuario. Seguidamente, se colocó la hembra apta para la reproducción (abdomen abultado) junto al macho 

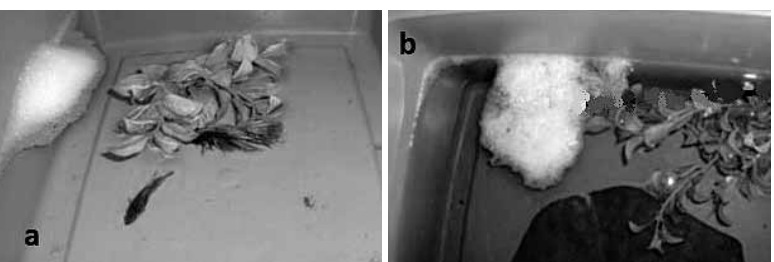

Figura 1. a) Cortejo del macho para la realización de la fecundación. b) Huevos fecundados ubicados en el colchón de burbujas.

para que éste inicie la danza de cortejo, la cual consistió en mover y ampliar sus ornamentos sexuales (los opérculos y la extensión de su aleta anal) alrededor de ella. Tras una persecución no exenta de golpes y mordeduras, la hembra lo aceptó. Esto sucedió cerca del nido de burbujas, donde el macho abrazó a la hembra y ejerció presión sobre su vientre haciendo que desove (extrucción). Acto seguido él liberó el semen fertilizando los huevos (Figura 1b).

Fecundación y cuidado parental. Esta fase comenzó inmediatamente después de la fertilización. La hembra fue retirada y el macho inició su cuidado parental subiendo los huevos uno por uno y pegándolos al nido. Los huevos caídos al fondo fueron recogidos y ubicados nuevamente en el nido (Figura 1b). Las hembras desovaron entre 60 y 85 huevos aproximadamente, estos eran de color blanco y no flotaban.

Incubación y eclosión. En este estadio los oocitos fertilizados se transformaron en embriones, anexados a la membrana coriónica y al disco germinal. Este último registró un tamaño de 529,39 $\mu \mathrm{m}$. El saco vitelino midió $679,43 \mu \mathrm{m}$ (Figura 2). La temperatura del agua de incubación fue de $27,8 \pm 1,5^{\circ} \mathrm{C}$ y un $\mathrm{pH}$ de $6,8 \pm 0,2$. La tasa de fecundación de los desoves realizados fue de $83 \pm 2,0 \%$. El tiempo transcurrido desde la fecundación hasta la eclosión fue de 36:13 h; durante este tiempo el embrión atravesó por los siguientes períodos: cigoto,
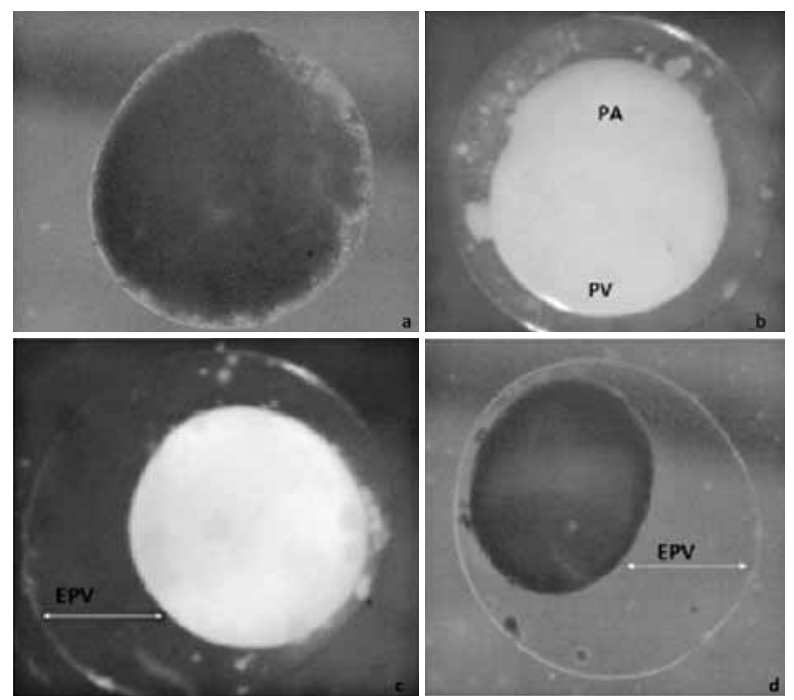

Figura 2. Estadio de cigoto. a) cigoto; b) estadio de una célula, $10 \mathrm{MPF}$, se observa el PA y PV; c) y d) cigoto, estadio de una célula, $12 \mathrm{MPF}$ y se observa el aumento del EPV. clivaje (blástula), gástrula, segmentación y organogénesis, eclosión y estado larval.

Período de cigoto. Luego de la fecundación e hidratación, el cigoto (Figura 2a) presentó un espacio perivitelino (Figura 2b) que duplicó en diámetro al embrión en desarrollo: Figura $2 \mathrm{~b}(\mathrm{EPV}=77 \mu \mathrm{m})$, Figura $2 \mathrm{c}$ $(\mathrm{EPV}=106 \mu \mathrm{m})$ y Figura $2 \mathrm{~d}(\mathrm{EPV}=149 \mu \mathrm{m})$; este estadio se observó aproximadamente entre los 10 y $12 \mathrm{MPF}$, en ese período de tiempo se produjo la segregación del blastodisco diferenciando un polo animal y uno vegetal (Figura 2b).

Período de clivaje (blástula). El clivaje se realizó después de la fecundación, aproximadamente a los 50 MPF, donde el huevo sufrió divisiones en el citoplasma (Figura 3). Se presentó un desarrollo de blástula temprana donde el blastodisco adoptó una forma ovalada diferenciando dos polos, por lo cual se reconoció que los huevos eran del tipo telolecítico, ya que acumulan vitelo en el polo vegetal de los mismos y presentan una división parcial o meroblástica durante los primeros estadios del desarrollo, con un EPV=93 $\mu \mathrm{m} ; \mathrm{ab}=512 \mu \mathrm{m}$, $\mathrm{AV}=592 \mu \mathrm{m}$ y $\mathrm{LV}=490 \mu \mathrm{m}$ (Figuras 3 y 4 ).

Período de gástrula. En el periodo de gastrulación (Figura 5), continuó el movimiento de epibolia, al que se sumaron los movimientos morfogenéticos de involución, convergencia y extensión, que llevaron finalmente a la generación de las diferentes capas germinales y
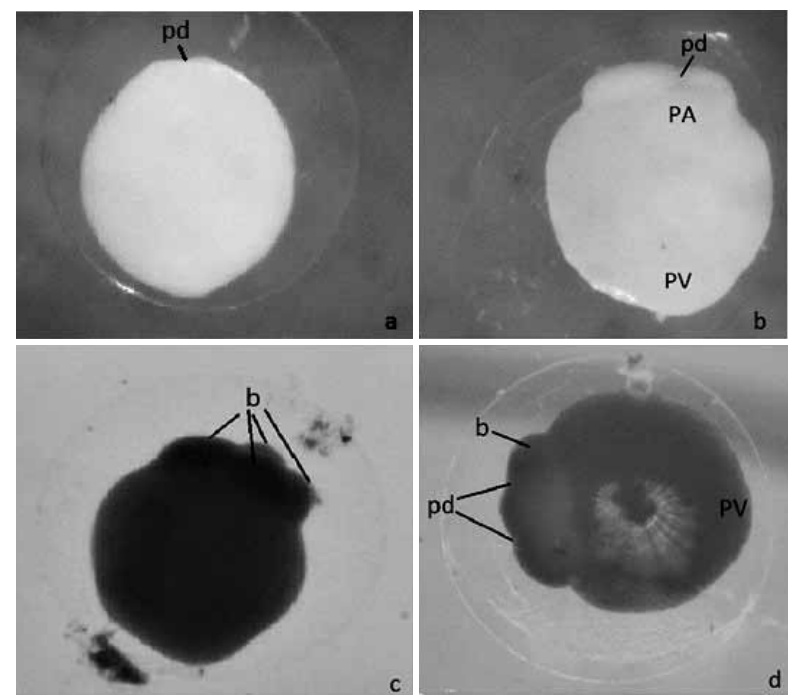

Figura 3. Estadio de clivaje. a) inicio del PD para la formación de $\mathrm{B}$; b) formación de 2 células; c) y d) formación de 4 células.
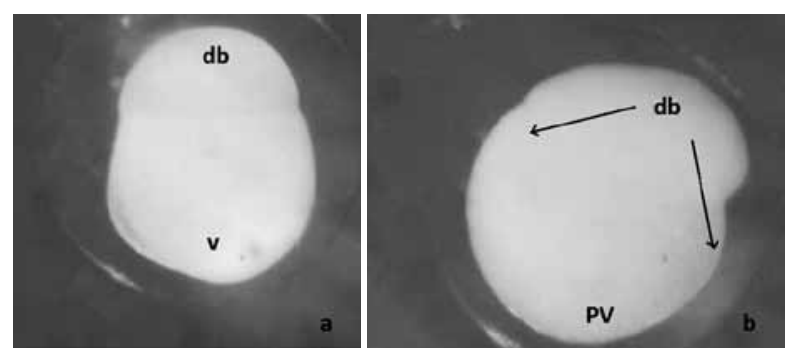

Figura 4. Estadio de blástula. a) Elevación del DB; b). Inicio del descenso del DB. 

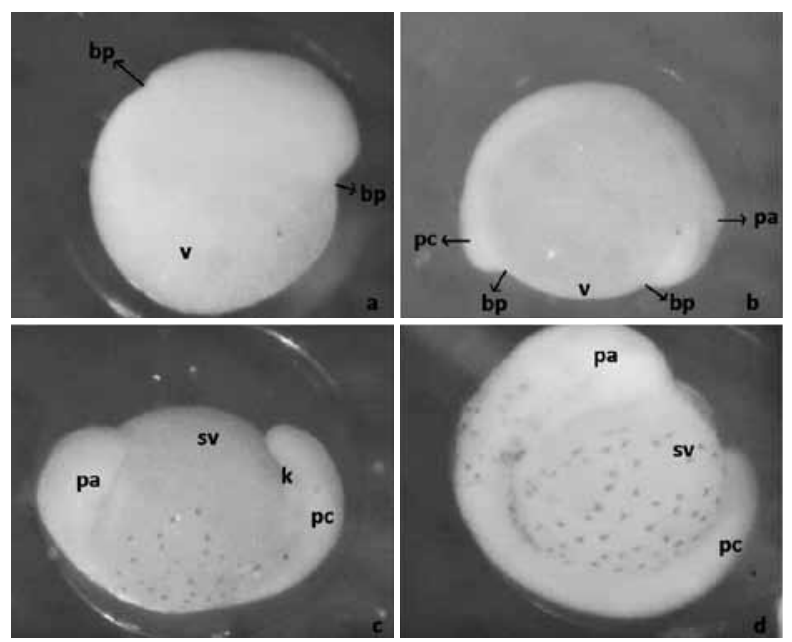

Figura 5. Estadio de gástrula. a) Epibolia del 30\% aproximadamente; b). Inicio de la formación del embrión; c) y d). Embrión en formación diferenciado antero-posterior (eje cráneo-caudal) en forma arqueada. Se observaBP, V, A, PC, K y SV.
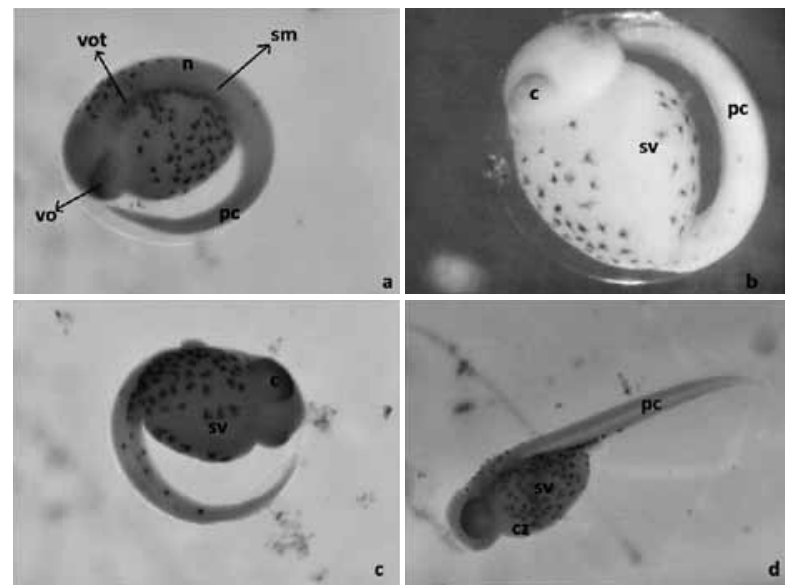

Figura 6. Estadios de organogénesis y eclosión. a) Organogénesis temprana, aparecen las VO y VOT; b). Embrión avanzado en forma arqueada, diferenciación total del eje cráneo-caudal, inicia desprendimiento de la cola y se observa el C; c) y d). Larva eclosionada. Se observa PC, SV y CZ.

ejes embrionarios, observándose que el cigoto presentaba una forma ovoide donde claramente se reconocía el anillo embrionario, el eje dorsoventral del embrión y la zona de evacuación, lo cual indicó la formación del eje anteroposterior del embrión. Todo este proceso abarcó un tiempo de 55 minutos, con un diámetro de vitelo de $530 \mu \mathrm{m}$, ancho de $612 \mu \mathrm{m}$ y largo de 646 $\mu \mathrm{m}$. Por su parte, la cabeza registró $216 \mu \mathrm{m}$ de ancho y 351,20 $\mu \mathrm{m}$ de largo.

Período de segmentación y organogénesis. El estadio de segmentación (Figura 5c y 5d) y organogénesis (Figura 6a y 6b) tardó aproximadamente 16 min seguido de la morfogénesis, la cual registró una duración de alrededor de 21 h. Se encontró un Lvo=89 $\mu \mathrm{m}$ y $A v o=50 \mu \mathrm{m}$ (Figura 5b); asímismo $L V=81,2 \mathrm{~mm}$, $\mathrm{AV}=80 \mathrm{~mm} ; \mathrm{DPO}=76,3 \mathrm{~mm} ; \mathrm{EE}=528 \mu \mathrm{m} ; \mathrm{LN}=490 \mu \mathrm{m}$;
$\mathrm{AN}=41 \mathrm{~mm} ; \mathrm{LC}=318,5 \mu \mathrm{m}, \mathrm{Lvo}=53,4 \mu \mathrm{m}$ y $\mathrm{Avo}=48,8$ $\mu \mathrm{m}$ (Figura 5d). Se observó la formación del tubo neural, se inició la somitogénesis y se produjeron las primeras diferenciaciones morfofisiológicas (aparición de los esbozos de los órganos primarios, aumento del tamaño del primordio de la cola, elongación del embrión y aparición de la vesícula óptica, observándose los primeros movimientos corporales (como latidos del corazón) y surgimiento de la vesícula de Kuppfer.

Período de eclosión y estado larvario. En la última etapa, que tardó unas $13 \mathrm{~h}$, se observó la eclosión y el estado larvario de $B$. splendens. En esta última fase se detectaron los inicios de la aleta caudal, tanto en la parte anterior como posterior, un vitelo de longitud más pronunciada, de $702 \mu \mathrm{m}$ y de grosor de menor tamaño. La cola registró una flexibilidad apropiada para su inicio (Figura 6c y 6d).

\section{DISCUSIÓN}

La descripción de las estrategias reproductivas utilizadas por $B$. splendens se basaron principalmente en características: morfológicas (policromía) ${ }^{24}$, ecológicas (coexistencia con otras especies, sobrevivencia con micrófitas y en superficies quietas, sin aireación, tolerancia de temperaturas entre 26 y $30^{\circ} \mathrm{C}$ y $\mathrm{pH}$ entre 7.0 y 7.5), y etológicas (cortejo, fertilización, fecundación y cuidado parental, incubación y eclosión) ${ }^{12}$, cualidades que permite su fácil reproducción y desarrollo en cautiverio.

El desarrollo de $B$. splendens desde la fecundación hasta la eclosión ${ }^{1}$, pasando por diversas etapas (cigoto, clivaje o blastulación, gástrula, segmentación y organogénesis, eclosión y estado larval), en esta investigación insumió una duración de 36,13 h. Otros autores reportaron lapsos similares, con una diferencia de 1,13 horas ${ }^{4}$, posiblemente debido a la temperatura utilizada. Asimismo, el lapso aquí obtenido fue menor que el reportado para el pez cebra (Danio rerio) ${ }^{16}$ (48 h), pero mayor que el registrado en el hibrido de Piaractus brachypomus y Colossoma macropomum, que fue de $12 \mathrm{~h}^{6}$. En el pez ángel (Pterophyllum scalare), la eclosión ocurrió 42,5 h post-fecundación, cuando la incubación se hizo a $28^{\circ} \mathrm{C}{ }^{31}$.

El período de clivaje abarca las primeras seis divisiones incluyendo la blastulación, donde el blastodisco -inicialmente deforme- inicia su organización y la evolución de sus bordes sobre la vesícula vitelina ${ }^{31}$, donde se diferencian dos polos, por lo que se reconoce que los huevos son del tipo telolecítico, similares a los reportados para Pseudoplatystoma fasciatum ${ }^{27}$, Danio rerio y Fundulus heteroclitus entre otros, ya que acumulan vitelo en el polo vegetal y presentan una división parcial o meroblástica durante los primeros estadios del desarrollo ${ }^{28}$.

Para este periodo algunos autores reportan tiempos entre 40 min y 1,33 $\mathrm{h}^{7,17,23,27}$. El lapso obtenido en el presente estudio (50 MPF), es bastante corto en comparación con el periodo reportado en otros peces como Brycon siebenthalae ${ }^{11}$, Danio rerio ${ }^{16}$ y Prochilodus lineatus $^{7}$. 
El período de gastrulación resultó similar al reportado para Prochilodus lineatus ${ }^{7}$ Danio rerio, Oryzias latipes, Fundulus heteroclitus, Barbus conchonius y salmónidos ${ }^{28}$, donde el elemento común entre estas especies y el $B$. splendens es que la gastrulación es un fenómeno concomitante a la epibolia que acontece a partir de un grupo de células localizadas en el polo animal del huevo telolecito (blástula) y durante la gastrulación, las células de la blástula experimentan una redistribución dramática para originar un embrión multilaminar con rudimentos de cabeza, tronco y cola ${ }^{34}$, generado a través de movimientos celulares altamente coordinados mediante los cuales, grupos definidos de células se internalizan desde la superficie formando un blastoporo y dando origen al endomesodermo ${ }^{28}$.

Los estadios de segmentación y organogénesis fueron similares al desarrollo embrionario descripto para Pseudoplatystoma fasciatum ${ }^{27}$ y Danio rerio ${ }^{19}$. Asimismo, aparece en B. splendens la vesícula de Kuppfer, una estructura transitoria que se presenta en el desarrollo de los teleósteos durante el período de segmentación ${ }^{7}$. La última etapa (eclosión y estado larvario) fue similar a la reportada para Brycon siebenthalae donde se observan los primordios de la aleta caudal, vitelo más pronunciado y cola más flexible ${ }^{11}$.

Los oocitos de peces de agua dulce contienen en su mayoría gran cantidad de vitelo (telolecito, al igual que en otros teleósteos) ${ }^{7,28}$, lo que conlleva la formación de embriones y larvas pequeñas. Este acontecimiento facilita la difusión de oxigeno por todo el cuerpo, cuando la presión molecular $\mathrm{O}_{2}$ dentro del embrión o de la larva, es mejor que fuera de sí mismos, haciendo que los gases (oxigeno, dióxido de carbono y amoníaco) se difundan a través de diversas membranas biológicas, incluyendo el corion ${ }^{11}$.

Embriones y larvas pelágicas usualmente no desarrollan órganos respiratorios especializados y generalmente es tardía la aparición de eritrocitos en plasma sanguíneo. Sin embargo, los peces laberinto pertenecientes a la familia Anabantidae (subfamilia Ctenopinae, genero Betta), presentan un órgano respiratorio adicional situado en las agallas, compuesto por numerosas láminas que permiten respirar aire fuera de la superficie del agua ${ }^{9}$. Esta característica fisiológica posibilita la respiración en aguas con temperaturas muy elevadas y poco oxígeno ${ }^{13}$.

En conclusión, se aportan datos que profundizan el conocimiento de las estrategias reproductivas y el desarrollo embrionario de $B$. splendens, que en condiciones de laboratorio insumen un lapso relativamente corto en comparación con otras especies, hallazgos que pueden contribuir a mejorar la reproducción de este pez con fines comerciales.

Agradecimientos. A la Universidad de la Amazonia por brindar la infraestructura necesaria para la investigación. Al estudiante del programa de Medicina Veterinaria y Zootecnia, Sergio Luis Oliveros, por su colaboración en las tareas realizadas.

\section{REFERENCIAS}

1. Almonacid C. 1996. Caracterización molecular de isoenzimas de treo-D-isocitrato NADP oxidoreductasa en Hyla labialis. Tesis de maestría, Pontificia Universidad Javeriana, Bogotá, $67 \mathrm{p}$.

2. Amano M, Yamnome T, Yamada H, Okuzawa K, Yamamori K. 2004. Effects of photoperiod on gonadotropin-releasing hormone levels in the brain and pituitary of underyearling male Barfin flounder. Fish Sci 70: 812-818.

3. Arboleda O, Duván A. 2005. Calidad del agua y mantenimiento de acuarios. Revista Electrónica de Veterinaria, www.veterinaria.org/revistas/redvet/n080505.html, Redvet VI: 8 .

4. Arboleda O, Duván A. 2006. Crianza y producción del betta (Betta splendens) para acuaristas no profesionales. Revista Electrónica de Veterinaria, www.veterinaria.org/ revistas/redvet/n040406.html, Redvet VII: 4.

5. Atencio V, Arabia RF, Aristizabal J. 2006. Desarrollo embrionario y larvario de Dorada Brycon sinuensis. Comunic. Científ. CIVA. On line: http://www.civa2006.org, p. $604-618$

6. Botero M, Fresneda A, Montoya F, Olivera A. 2004. Descripción del desarrollo embrionario de zigotos híbridos obtenidos por el cruce de machos de Cachama Blanca (Piaractus brachypomus) y hembras de Cachama Negra (Colossoma macropomum). Rev Col Cienc Pec 17: 8.

7. Botta P, Sciara A, Arranz S, Murgas L, Pereira G, Oberlender G. 2010. Estudio del desarrollo embrionario del sábalo (Prochilodus lineatus). Arch Med Vet 42: 109-114.

8. Carrillo Estévez MA et al. 2009. La reproducción de los peces: Aspectos básicos y sus aplicaciones en la acuicultura. Edit. Publicaciones Científicas y Tecnológicas de la Fundación Observatorio Español de Acuicultura (OESA), Madrid, $719 \mathrm{p}$.

9. Castro AD. 2001. A look at the Anabantoids. A new series on these versatile fishes. Aqu Fish Magaz (AFM) 1: 01.

10. Chaves C, Chacón L, Lozada J, Motta PA, Murcia B. 2011. Evaluación de la reproducción inducida de cachama blanca (Piaractus brachypomus) con acetato de buserelina. Vet Zootec 6: 47-55.

11. Clavijo AJ, Arias CJ. 2004. Avances en el estudio de la embriología del yamú, Brycon siebenthalae (Pisces: Characidae). Dahlia Rev Asoc Col Ictiol 7: 37-48.

12. Fosgren E, Reynolds JD, Berglund A. 2002. Behavioral ecology of reproduction in fish. In: Handbook of fish biology and fisheries (Hart PJ, Reynolds JD ed.), vol.1, Blackwell Publishing, Oxford, p. 225-247.

13. García L. 2008. Efecto de la especie y color de los oponentes en la agresividad de los machos de Betta splendens (Regan, 1910). An Univ Etol 2: 37-42.

14. Guevara E. 1997. Estudio embrionario y larval microscópico e histológico de Betta splendens (Regan, 1909). Tesis de Maestría, Pontificia Universidad Javeriana, Bogotá, 140 p.

15. Hellqvist A, Bornestaf C, Borg B, Schmitz M. 2004. Cloning and sequencing of the FSH-b and LH-b subunit in the three-spined stickleback, Gasterosteus aculeatus, and effects of photoperiod and temperature on LH- $\beta$ and $\mathrm{H} \beta$ mRNA expression. Gen Comp Endocrinol 135: 167-174. 
16. Kimmel CB, Ballard WW, Kimmel SR, Ullmann B, Schilling TF. 1995. Stages of embryonic development of the zebrafish. Develop Dynam 203: 253-310.

17. Maestre-Serrano R, Guevara E, Colmenares I, Pachón E. 2008. Expresión y determinación de la actividad enzimática de las isoenzimas de L-Lactato-NAD-oxido-reductasa en el desarrollo embrionario temprano de Betta splendens (Regan, 1909). Universitas Scientiarum (Bogotá), 13: 11-20.

18. Miranda LA, Strüssmann CA, Somoza GM. 2009. Effects of light and temperature conditions on the expression of GnRH and GtH genes and levels of plasma steroids in Odontesthes bonariensis females. Fish Physiol Biochem 35: 101-108.

19. Moreno MF. 2013. Mantenimiento en el laboratorio del pez cebra (Danio rerio). Tesis de Pregrado, Universidad del País Vasco, España, 26 p.

20. Moser HG. 1996. The early stages of fishes in the California Current Region, Ed. Allen Press, Kansas, USA, 1505 p.

21. Nakatani K, Agostinho A, Baumgartner G, Bialetzki A, Vanderlei P, Cavicchioli M, Pavanelli C. 2001. Ovos e larvas de peixes de agua doce: desenvolvimento e manual de identificacao. Publ. Conselho Editorial, Maringá, 378 p.

22. Navarro MJ. 2007. Selección sexual en guppys (Poecilia reticulata, Pisces: Poeciliidae). An Univ Etol (Univ. Palmas Gran Canaria, España) 1: 80-84.

23. Ninhaus A, Foresti F, Azevedo A. 2006. Structural and ultrastructural analysis of embryonic development of Prochilodus lineatus (Characifirme, Prochilodontidae). Zygote 14: 217-229.

24. Oliveira RF. 2005. Neuroendocrine mechanisms of alternative reproductive tactics in fish. Fish Physiol 24: 297-357.

25. Ortiz F, Mejía A, Luz M, Acero PA. 2005. Description of eggs and early larval stages of the frog fish Antennarius striatus (Shaw, 1794) in captivity, with notes on its mechanism of reproduction. Rev Biol Mar \& Ocean 40: 23-31.

26. Ortiz VM. 2007. El pez betta, uno de los peces consentidos. On line: www.acuariolatino.com, p. 6.
27. Padilla P, Alcántara F, Bocanegra R, Ismiño R. 2001. Reproducción inducida de la doncella Pseudoplatystoma fasciatum y desarrollo embrionario-larval. Folia Amazónica 12: 1-2.

28. Pereiro González L. 2008. Análisis morfogenético de la gastrulación en peces teleósteos anuales del genero Austrolebias. Tesis Doctoral, Univ. de Chile, 147 p.

29. Pérez, LD, Peñaranda S, Dufour S, Baloche S, Palstra AP, Van Den Thillart GE, Asturiano JF. 2011. Influence of temperature regime on endocrine parameters and vitellogenesis during experimental maturation of "European eel" (Anguilla anguilla) females. Gen \& Comp Endocr 174: 51-59.

30. Petrovicky I. 1990. La gran enciclopedia de los peces de acuario, 1ra.ed., Edit. Susaeta, Madrid, 493 p.

31. Radael MC, Vázquez JM, Solis LD, Mattos C, Cardoso LD, Souza JH, Corrêa ML, Oliveira FV, Andrade DR. 2013. Desarrollo embrionario del pez ángel (Pterophyllum scalare). Arch Latin Prod Anim, 7 p. www.alpa.org.ve/ojs. index/php

32. Rosas J, Mata E, Velasquez A, Cabrera T. 2008. Desarrollo embrionario-larval del pez tropical Hemirhamphus brasiliensis (Beloniformes: Hemirhamphidae) a partir de huevos recolectados del medio natural. Rev Biol Trop 56: 1449-1458.

33. Saborido RF. 2008. Ecología de la reproducción y potencial reproductivo en las poblaciones de peces marinos. Public. Instit Investig Marinas (CSIC), Universidad de Vigo. España, 71 p.

34. Solnica KL. 2005. Conserved patterns of cell movements during vertebrate gastrulation. Curr Biol 15: R213-228.

35. Vargas Y, Yie T. 2003. Descripción, abundancia y distribución de la comunidad ictioplanctónica presente en el humedal-laguna Navío Quebrado (...), Caribe colombiano. Tesis Biol.Mar., Univ.J.T.Lozano, Santa Marta, Colombia, 80 p. 